\title{
POLITICAS PARA EL ANDAMIAJE DE TRAYECTORIAS DE ESTUDIANTES EN LA ESCUELA SECUNDARIA EN LATINOAMÉRICA: ESTRATEGIAS PARA GARANTIZAR EL DERECHO A LA EDUCACIÓN EN SOCIEDADES DESIGUALES
}

\author{
Pablo Daniel García* \\ Universidad Nacional de Tres de Febrero, Argentina \\ pgarcia@untref.edu.ar
}

Recibido: 13/08/2019 Aceptado: 30/11/2019

\begin{abstract}
Resumen
El presente artículo expone los resultados de una investigación que toma como objeto de estudio las políticas para el andamiaje de trayectorias escolares en la escuela secundaria en Latinoamérica. La investigación se basó en un estudio basado en una lógica cualitativa, realizada con el Método Comparativo Constante. El trabajo de campo que se considera en este artículo incluyó una selección de instituciones educativas que se ubican en contextos de alta vulnerabilidad social en cinco países de la región: Bolivia, Chile, Perú, Paraguay y Uruguay. Se realizó un relevamiento de las problemáticas vinculadas a las trayectorias escolares de los estudiantes de nivel medio que viven en contextos de vulnerabilidad y un mapeo y análisis de las intervenciones que se realizan desde la política educativa para el andamiaje de dichas trayectorias. Este artículo incluye, además de la presentación de algunos elementos teóricos y metodológicos que guiaron la investigación, una breve contextualización histórica de las políticas socioeducativas en la región y una presentación de algunas tendencias en relación al análisis de diferentes casos de países. Se analizan las estrategias mapeadas en particular considerando dos pares de categorías que permiten analizar las lógicas de intervención desarrolladas: focalización-universalismo e intervenciónautonomía.
\end{abstract}

Palabras claves: Política educativa - Trayectoria escolar - Escuela secundaria - Latinoamérica Derecho a la Educación.

\footnotetext{
Abstract

This article presents the results of an investigation that takes as an object of study the policies for the scaffolding of school trajectories in secondary school in Latin America. The research was based on a study based on qualitative logic, carried out with the Constant Comparative Method. The field work included a selection of educational institutions that are located in contexts of high social vulnerability in five countries of the region: Bolivia, Chile, Peru, Paraguay and Uruguay. A survey was carried out of the problems related to the school trajectories of middle-level students living in vulnerable contexts and a mapping and analysis of the interventions that are carried out from the educational policy for the scaffolding of these trajectories. This article includes, in addition to the

*Doctor en Educación, Magister en Políticas y Administración de la Educación, Licenciado y Profesor en Ciencias de la Educación. Profesor e Investigador en la Universidad Nacional de Tres de Febrero. Coordinador del Programa de Posgrados en Políticas y Administración de la Educación de la Universidad Nacional de Tres de Febrero.
} 
presentation of some theoretical and methodological elements that guided the research, a brief historical contextualization of socio-educational policies in the region and a presentation of some trends in relation to the analysis of different country cases. The mapped strategies are analyzed in particular considering two pairs of categories that allow analyzing the intervention logics developed: focus-universalism and intervention-autonomy

Keywords: Educational Policy - School Career - Secondary School - Latin America - Right to Education.

\section{Introducción}

La situación problemática a partir de la cual se organizó la investigación en la que se basa el presente artículo fue la exclusión educativa en Latinoamérica, en particular referida a los jóvenes provenientes de sectores vulnerables que asisten a la educación de nivel medio. El objeto de estudio de esta investigación fueron las políticas y estrategias para mejorar las trayectorias escolares de los estudiantes que se desarrollan en las instituciones educativas de nivel medio de Latinoamérica que trabajan en contextos de vulnerabilidad social.

Las trayectorias escolares dan cuenta de los recorridos que realizan los sujetos en el sistema escolar. Resulta pertinente recuperar la distinción de Terigi (2009) entre trayectorias teóricas y reales. Las trayectorias teóricas expresan recorridos de los sujetos en el sistema educativo que siguen la progresión lineal prevista por éste en los tiempos marcados por una periodización estándar. Las trayectorias reales de los sujetos pueden tener itinerarios frecuentes o probables, coincidentes o próximos a las trayectorias teóricas. En las trayectorias escolares reales es posible identificar itinerarios que no siguen los caminos ideales pensados y que toman modos heterogéneos, variables y contingentes (Terigi, 2007). Estos fenómenos particularmente son importantes de considerar al momento de analizar los recorridos por el sistema educativo de los estudiantes que provienen de contextos vulnerables.

Durante mucho tiempo se supuso el origen individual de las dificultades en el tránsito por el sistema educativo. Este modelo de responsabilidad individual generó mecanismos de diagnóstico, derivación y recuperación de los niños considerados en dificultad y consecuentes procesos de etiquetamiento y segregación, que buscaban encontrar el "fracaso" en fallos cognitivos o bien en la condición de origen de los estudiantes (Terigi y Baquero, 1997). Si bien se sostuvo el origen individual de las dificultades en el sistema educativo, en Latinoamérica en los años '80 se produce un giro en las formas de intervención sobre el problema a partir de empezar a considerar las condiciones sociales asociadas al fracaso escolar. Los procesos de recuperación democrática que tuvieron lugar en muchos países de la región fueron acompañados por una vasta reflexión sobre el fracaso escolar y la mirada comenzó a ponerse en las condiciones de desigualdad social (Kachinovsky y Gabbiani, 2013). Evitar el fracaso escolar comenzó a ser asunto de políticas sociales de atención a la niñez y la juventud y de políticas educativas compensatorias de las desigualdades (Terigi, 2009).

Ahora bien, los avances en la investigación educativa sobre estas temáticas hoy en día permiten comprender que la desigualdad social es parte de la explicación del fracaso educativo, pero no lo explica totalmente. Si bien resultan de vital importancia las ayudas sociales para andamiar el paso por la escolarización de los sectores más pobres, investigaciones recientes en el campo demuestran que existen barreras u obstáculos al interior de la propia institución escolar que derivan en situaciones del Ilamado fracaso escolar. Siguiendo a Serulnikov (2008), hay responsabilidad institucional de las escuelas y del sistema educativo en general en la propia sentencia de fracaso. Las escuelas comienzan a asumir la responsabilidad de que niños, niñas y jóvenes no aprendan e incluso, a veces, que no concluyan el ciclo escolar. A la vez, en los últimos años, las categorías de inclusión y exclusión han ganado terreno para explicar estos fenómenos que suceden al interior de las instituciones escolares. Cada vez más se pone el foco en prácticas y acciones desarrolladas al interior de las propias instituciones para comprender los resultados que los estudiantes tienen. Las posibilidades de los jóvenes y adolescentes de aprender en la escuela dependen cada vez menos de las capacidades que supuestamente portan (o les faltan) y más de las particularidades de la situación educativa y de las condiciones pedagógicas en que tiene lugar su escolarización (Terigi, 2009). Por lo cual, problemáticas tales como repitencia, sobreedad, ausentismo, abandono, están asociadas más que al fracaso escolar individual, a persistentes puntos críticos del sistema educativo. La inclusión educativa, condición para el pleno cumplimiento del derecho a la educación, supone que se haga efectiva la asistencia a la escuela de todos los que se encuentran en edad escolar, que los centros escolares garanticen la calidad del servicio brindado, que la formación sea compartida con otros sin que por ello anule las singularidades y la cultura local, ni codifique como única cultura autorizada la de algunos sectores sociales (Terigi, 2009). La investigación realizada tuvo como objetivo el análisis de las políticas que se desarrollan en un grupo de países de Latinoamérica para el logro de trayectorias continuas y completas en el paso de los estudiantes de sectores vulnerables por la escuela secundaria.

En términos metodológicos, la investigación en la que se basa este artículo se organizó a partir de una lógica cualitativa (Achili, 1992), a partir de la construcción de un trabajo basado en la inducción analítica para la búsqueda de la generación de teoría y la comprensión de la realidad y tuvo como herramienta principal el Método Comparativo Constante diseñado por Strauss y Corbin (1991). Se optó por Latinoamérica como campo de trabajo para tener una mirada de la diversidad de la región. La aspiración 
fue lograr una mirada que transite por diversidad de contextos para mostrar la complejidad del fenómeno estudiado en una selección de países de la región. El trabajo de campo se basó en entrevistas a directores y equipos de gestión de instituciones de nivel secundario. Quedaron incluidas en el estudio 38 instituciones educativas del nivel medio de 7 países de la región ( 6 de Chile, 5 de Bolivia, 5 de Perú, 6 de Ecuador, 5 de Paraguay, 7 de Argentina y 4 de Uruguay), visitadas en 2015 y 2016. El abordaje de campo en cada institución supuso una estadía breve (de 2 a 3 días) a fin de combinar diversas técnicas de recolección empírica. Las entrevistas realizadas fueron complementadas con relatos en profundidad de experiencias de la vida institucional, lecturas de documentación generada por la propia institución vinculada a acciones y/o proyectos de inclusión educativa y análisis de normativa oficial que se vincula a las políticas educativas consideradas. En este artículo se consideran las experiencias seleccionadas en Chile, Bolivia, Paraguay, Perú y Uruguay.

\section{La intervención en lo socioeducativo desde comienzos del milenio: breve historización de las políticas en la región}

Los años noventa en Sudamérica han sido tiempos de cambios en lo que se refiere a la configuración de los Estados Nacionales y de su accionar, las políticas públicas. Es posible afirmar que la década del noventa fue escenario de la consolidación de la transformación estructural de la relación estado y sociedad, que es expresión de un profundo cambio político, cultural y económico en la región. Dicha transformación ha sido denominada neoliberal y está asociada al inicio de un nuevo ciclo de acumulación capitalista con procesos de reajuste y sobre todo de reforma del estado en los países latinoamericanos, que se bosquejaron con las dictaduras de los setenta y se expresaron plenamente en los 90. En estos años, el Estado transitó complejos procesos de cambio asociado a las ideas de "desaparición del estado", "retiro" o "reducción". Sin embargo, a pesar de que cambió, el estado no desapareció, sino que reconfiguró su rol. Esto se desarrolla en la región en simultáneo con un abrupto incremento de la pobreza de la población, y agravando tal situación, una indudable polarización de la desigualdad en la distribución de los recursos económicos y del acceso a los bienes culturales. En este contexto aparece un replanteamiento de la relación estratégica entre el progreso y la educación. Se instala entonces como consigna para la planificación de las políticas educativas de la década la idea de "Educación para Todos" y comienza un proceso arduo de creación de consensos al interior de los Estados para establecer políticas de reforma educativa. Se inauguraba así una nueva ola reformista en Sudamérica, para la cual resultó fundamental el apoyo del Banco Interamericano de Desarrollo a través de la creación del PREAL Programa de Promoción de la Reforma Educativa en América Latina- (Gajardo, 1999). Ejemplos de políticas de esta época pueden considerarse el "Programa 900 Escuelas en Chile" o el "Plan Social Educativo" en Argentina. Ambos, dan cuenta de la estrategia típica en la época para el de trabajo con poblaciones vulnerables en la región: para seleccionar beneficiarios, la focalización a partir de procesos de discriminación positiva y luego, una lógica de provisión (provisión de textos y materiales de instrucción, bibliotecas, otros recursos), extensión de la jornada escolar, incremento de horas de clase y en menor medida, programas de innovación pedagógica y de reforma curricular (Gajardo, 1999). Los mencionados planes constituyen ejemplos paradigmáticos de políticas focalizadas compensatorias como forma de intervención estatal. Se proponen compensar las desigualdades de origen de los estudiantes concentrando los recursos en la "población objetivo" (los más pobres entre los pobres) y se fundan en el principio de equidad, concebida como no dar lo mismo a quienes no son iguales (Feldfeber y Gluz, 2011).

Años después de la primera implementación de las reformas, caracterizadas por un anti-estatismo radical, surgen nuevas orientaciones. Con el "Informe sobre el Desarrollo Mundial" de 1997 el Banco Mundial expresa un giro con respecto a las reformas de los noventa: el estado deja de ser causante de todos los obstáculos para el desarrollo y se reconocen en él, ventajas. Se inicia a partir de entonces una revalorización de lo estatal en un sentido general y de sus capacidades e instituciones. Así, el escenario latinoamericano que se esboza a partir del 2000, cambia con la presencia de varios gobiernos en Latinoamérica caracterizados como progresistas o de centroizquierda, va a implicar ciertos rasgos en lo que respecta a continuidades y rupturas con respecto a las reformas de los 90 . Si durante gran parte de las décadas de los ochenta y noventa, la planificación estatal fue reemplazada por programas o proyectos específicos, muy limitados, con financiamiento internacional, a principios del nuevo milenio, con una mirada crítica a este modelo, se volvía a repensar el rol del Estado en la planificación. La planificación recupera su rol en la construcción de políticas educativas. La propuesta de un modelo renovado de planificación, más dinámico, consiste en asumir metodologías que tengan en cuenta los constantes cambios y la rapidez de los procesos sociales y económicos (Fernández Lamarra, 2006).

Aparecen entonces a comienzos de los años 2000 grandes planes nacionales que tienen como objetivo alinear la planificación educativa con los objetivos de la "Educación para todos". El "Proyecto Educativo Nacional, políticas y metas prioritarias 2006-2011" de Perú, el "Plan Nacional Educación para Todos, Período 2003-2015" de Ecuador o el Plan Educacional Ñandutí, del Paraguay pueden ser algunos ejemplos de estos grandes planes nacionales. Estas "macroplanificaciones" de política educativa son construidas desde el nivel central de conducción del sistema (los ministerios), a veces con consulta y/o procesos de consenso con jurisdicciones y organizaciones de la sociedad civil, donde se fijan metas para el desarrollo de cada sistema educativo, objetivos, estrategias, prioridades de financiamiento, entre otras cuestiones. Estos grandes planes, en general, permiten poner "en agenda" temáticas, dar cuenta de las prioridades del periodo y garantizar algunos acuerdos políticos. 
A la vez, en paralelo a estos planes nacionales desarrollados en cada país de la región, han llevado a cabo procesos de institucionalización de la gestión de políticas para sectores vulnerables en el ámbito de los ministerios del sector. Se conforman así equipos técnicos específicos dentro de la estructura ministerial y responsables políticos de atender las problemáticas vinculadas a la pobreza / desigualdad social. Por ejemplo, se ha creado en Perú, en el ámbito del Ministerio de Educación, la Dirección Nacional de Becas y Crédito Educativo; en Chile la Junta Nacional de Auxilio Escolar y Becas (JUNAEB) o en Ecuador la Subsecretaría de Calidad y Equidad Educativa, de la cual depende la Dirección Nacional para la Democracia y el Buen Vivir. Esta institucionalización contribuye a la continuidad de las políticas que desde estas dependencias se desarrollan y su interconexión con otras áreas del estado.

\section{Las estrategias para la mejora de las trayectorias escolares en las escuelas secundarias en la región}

La caracterización realizada en los párrafos precedentes permite conocer las bases y algunos de los debates sobre las que se asientan las políticas que actualmente se desarrollan en la región para garantizar el Derecho a la Educación, en particular de los sectores más vulnerables. A continuación, se presenta un análisis de las estrategias implementadas en un grupo de países de la región para andamiar las trayectorias escolares en la escuela secundaria. Cada Estado, a partir de las políticas que desarrolla, genera múltiples intervenciones que dan cuenta de sus prioridades y de sus preocupaciones. Se consideran en particular los casos de Chile, Bolivia, Paraguay, Uruguay y Perú.

El trabajo de campo realizado en los Liceos de Chile y su posterior análisis permite dar cuenta de algunos rasgos de las políticas desarrolladas en dicho país para el andamiaje de las trayectorias escolares en la escuela secundaria. Se trata de una lógica de intervención con un fuerte componente de focalización. La misma se manifiesta, en primer lugar, en la provisión de diferentes tipos de becas organizadas según diferente población destinataria y una clasificación previa de todos los estudiantes como "prioritarios" o "vulnerables". Aquí el "poder de nombramiento" de la escuela se manifiesta en plenitud (Kaplan, 2006). El complejo andamiaje conceptual del sistema de asistencia escolar en Chile se organiza a través de una particular forma discursiva con su respectivo sistema de clasificación y nominación. La sentencia a algunos estudiantes como vulnerables, y a otros como prioritarios son expresiones de este tipo de operaciones del lenguaje sobre la realidad social. Sería de mucho interés analizar en qué medida la evaluación y calificación que realizan los docentes no queda atrapada en estas categorizaciones. Resulta de interés destacar que las distinciones a partir de las cuales se establece una división de los estudiantes incorporaron ya desde su génesis cualidades y virtudes no exclusivamente escolares sino también sociales, familiares, y de otras esferas (Kaplan, 2006). Con la misma lógica de nombramiento/clasificatoria se designa a algunos liceos los cuales, por el tipo de población a la que atienden, quedan en el marco de la denominada Ley SEP (Ley de Subvención Escolar Prioritaria). A los liceos que concentran gran cantidad de estudiantes vulnerables y prioritarios se los denomina focalizados y es muy sencillo encontrar la nómina para ciudad de cada uno de ellos. Resulta importante considerar este aspecto y analizar en qué medida esto no genera una situación de segregación/estigmatización hacia estas instituciones.

El Programa de Alimentación Escolar (PAE) de Chile funciona con la misma lógica de focalización: solo pueden acceder los estudiantes focalizados de acuerdo a su condición de vulnerabilidad. Actualmente solo participan del PAE los estudiantes de familias que pertenecen al $60 \%$ más vulnerable de la población o con mayor desventaja socioeconómica según el "Registro Social de Hogares". Con el "índice de vulnerabilidad" de estudiantes por establecimiento, la infraestructura disponible y el dato de su matrícula, la JUNAEB determina la cantidad y tipo de raciones que recibirá el colegio. Así, otra operación de nombramiento aparece: estudiantes que reciben el almuerzo escolar y estudiantes que no acceden al mismo.

Resulta interesante agregar también que con la misma lógica de focalización se desarrollan otras dos intervenciones muy importantes en términos del cumplimiento efectivo del Derecho a la Educación en Chile: el Programa de Integración Escolar (PIE) -para estudiantes con discapacidad- y el Programa de Educación Intercultural bilingüe. El Programa de Educación Intercultural Bilingüe, por su lado, funciona exclusivamente en las escuelas y liceos donde estudian niños y niñas de los pueblos indígenas del país. La focalización en un determinado tipo de instituciones aparece nuevamente, con las limitaciones que esto supone. En lugar de promover un sistema educativo capaz de incluir a los pueblos originarios, generando acciones de sensibilización e innovación curricular en todas las instituciones del país, específicamente se concentra en aquellas instituciones a las que asisten estos sujetos.

Podría sintetizarse la estrategia de Chile como un modelo altamente focalizado con un componente fundamental de provisión de recursos y un escaso desarrollo de modelos pedagógicos alternativos. En el modelo chileno además el desarrollo de propuestas institucionales de inclusión/retención tiene un rol central, pero con un alto componente de control de parte de instancias centrales de la administración educativa a través de la provisión de fondos y su rendición de cuentas.

\section{Bolivia: la transformación de la escuela secundaria centrada en la comunidad}

Ante un contexto histórico signado por la dominación de una minoría mestizo-criolla durante los siglos XIX y XX, el ascenso de Evo Morales en 2005 a la presidencia de Bolivia representó un cambio radical tanto en términos políticos como simbólicos. La llegada al poder de un presidente indígena proveniente de las filas del sindicalismo campesino inició un nuevo camino hacia la integración y el respeto a la 
diversidad (Efrón, 2013). En el ámbito educativo, este cambio se plasmó con la sanción en diciembre de 2010 la Ley de la Educación № 70 Avelino Siñani-Elizardo Pérez. Su nombre explicita las bases sobre las que se pretendía fundamentar la configuración de un nuevo sistema educativo: la experiencia de la Escuela-Ayllu de Warisata de 1931-1937. Esta experiencia se basó en el reconocimiento de las tradiciones de las comunidades indígenas y se convirtió en un modelo de lucha contra la omisión, abuso y opresión. Sus principios fueron la complementariedad y reciprocidad entre la comunidad y la naturaleza (Mamani Cussy, 2011). La nueva legislación boliviana busca recuperar la orientación de Warisata, en el caso del nivel medio, a través de la redefinición de la educación secundaria como Educación Secundaria Comunitaria y Productiva. La Escuela Secundaria Comunitaria y Productiva implica el lugar el desarrollo de un modelo de gestión escolar comunitario, que se ejecuta a partir de la participación democrática y de la construcción de consensos, donde la comunidad está comprometida con la gestión educativa para fortalecer el desarrollo de sus capacidades, habilidades y valores comunitarios en convivencia armónica con la naturaleza. Además, se plantea el desarrollo de una propuesta productiva en el marco escolar, que desarrolle conocimientos en relación directa con el trabajo y la producción material e intelectual científica y tecnológica. Ambas dimensiones (la comunitaria y la productiva) están vinculadas dado que se espera que el Proyecto Socio-productivo que arma cada institución educativa surja de un diagnóstico y trabajo con un alto grado de implicación de la comunidad. Se espera que la comunidad de referencia de cada institución educativa participe en la organización del trabajo para elaborar el proyecto, en el proceso de análisis e identificación de los problemas, en las necesidades sobre los que va a trabajar el proyecto, en la definición del Plan de Acción y por último la redacción y socialización del documento del proyecto. El componente participativo hace único a cada proyecto socio productivo.

La transformación de la escuela secundaria en Bolivia supone algunas variaciones del régimen académico muy notorias que apuntan al sostenimiento de trayectorias escolares de los estudiantes, en particular aquellas referidas a la evaluación de los aprendizajes. Se buscó un cambio radical con respecto a la evaluación, tradicionalmente enfocada en el aprendizaje de contenidos de manera memorística y en la capacidad de los estudiantes de retener información. También en este sentido se busca romper con la auto-referencialidad de la escuela y con la lógica meritocrática que trae aparejada la medición. El nuevo modelo de evaluación no la limita a la mera medición de la acumulación de conocimiento y actitudes centradas en la escuela como esfera separada del contexto más amplio del que forma parte. Otro aspecto sustancial de la transformación educativa en Bolivia se refiere al cambio curricular. A partir de la Ley de la Educación "Avelino Siñani-Elizardo Pérez" por primera vez en la legislación educativa boliviana se incorpora la importancia de la cosmovisión, conocimientos y saberes de los pueblos originarios como base de la educación nacional (Cajías de la Vega, 2013). A fin de considerar su influencia en las trayectorias escolares y particularmente en la experiencia escolar de los estudiantes, resulta de interés que los principios sobre los que se construye el nuevo marco curricular para la Educación Secundaria Comunitaria y productiva aspiran a la concreción de una educación intracultural, intercultural y plurilingüe. Este nuevo tipo de abordaje educativo se realiza a partir de recuperación de saberes, conocimientos y lenguas propias de las naciones indígenas, originarias y campesinas, promoviendo su vínculo con otras culturas.

Vale destacar que todas las intervenciones identificadas en las unidades educativas visitadas en Bolivia son universales en el sentido de que surgen de políticas educativas pensadas para todas las instituciones del nivel. En lo que respecta a la provisión de recursos para los estudiantes, apenas incluye el Bono Juancito Pinto, un bono de ayuda que se entrega a todos los estudiantes de un rango etario más allá de su situación económica familiar. Del mismo modo, todos los estudiantes son beneficiados por igual por el Programa de Alimentación. Podría pensarse a Bolivia a partir de una lógica de intervención donde predominan las intervenciones universales, que abarcan a todas las instituciones del nivel por igual y con el foco puesto en la transformación curricular e institucional de las unidades educativas, y con baja transferencia de recursos directos a los estudiantes. Se observa así mismo la asignación de un lugar estratégico destacado a las comunidades locales.

\section{Perú: el incipiente desarrollo de políticas para la mejora de las trayectorias escolares}

A partir del análisis de los testimonios recolectados en el trabajo de campo, en el caso de Perú la preocupación por el sostenimiento de trayectorias escolares no aparece como una problemática priorizada en las instituciones. A nivel de política educativa aparece un programa principal que concentra las innovaciones en el nivel secundario: la Jornada Escolar Completa (JEC). Es posible considerar este programa como planificado para lograr una cobertura universal en el nivel, pero con una aplicación progresiva, por lo cual, se enmarca dentro de los denominados "programas transversales" (Jacinto y Freytes Frey, 2004). En el 2015 el programa inició con una aplicación a 1000 colegios de gestión pública y en 2016 se extendió a otros 600 (de un total de casi 6000 en todo el país, que esperan cubrirse en su totalidad para el 2021). El programa JEC incrementa en 10 horas pedagógicas semanales el horario escolar, aumentando el tiempo de instrucción en áreas como Matemática, Inglés, Educación para el Trabajo, entre otras. Incluye acompañamiento al estudiante a través de un sistema tutorial y de reforzamiento pedagógico. Del mismo modo, brinda a docentes herramientas como cuadernillos sobre unidades didácticas y capacitaciones para integrar el uso de tecnologías al proceso de enseñanza y aprendizaje. Supone además una nueva organización de los tiempos escolares dado que además de incrementar la cantidad de horas del horario escolar, el tiempo pasa a ser destinado a otras funciones: los profesores deben organizar su horario incluyendo, además de las horas destinadas a las clases, horas 
destinadas a la planificación colegiada de las asignaturas, atención a estudiantes y padres de familia, revisión de materiales, entre otras actividades.

La JEC incorpora un uso innovador de los espacios escolares dado que los estudiantes se movilizan por diferentes aulas a lo largo de la jornada (las aulas ya no se asignan a los grupos de estudiantes sino a las disciplinas por lo cual las actividades de un mismo grupo a lo largo del día varían de aula 3 o 4 veces). Otro de los dispositivos que incluye la JEC para el acompañamiento de las trayectorias escolares de los estudiantes es el de la "Acción Tutorial Integral" (ATI). Para su implementación, se ha ampliado la hora de tutoría grupal a dos horas y se ha previsto un tiempo y espacio para realizar la tutoría individual. Asimismo, se han seleccionado docentes para que desarrollen la función de Coordinadores de Tutoría, y para el desarrollo de sus actividades se le ha asignado dieciocho horas para labores de acompañamiento a docentes y tutores. El espacio ATI, que incluye la asignación de un horario específico en el cronograma escolar, se encuentra pautado minuciosamente desde el nivel central (con cada una de las clases ya planificadas de antemano para abordar temáticas especificas). Esto le resta autonomía a un espacio que podría adaptarse a las diferentes situaciones de cada comunidad educativa. Además, el modelo de JEC incluye los cargos de psicólogo y/o trabajador social para trabajar con la comunidad educativa.

Un aspecto a destacar de las estrategias identificadas en Perú para la mejora de las trayectorias escolares es la ausencia de políticas de provisión de recursos directos a los estudiantes para compensar las desigualdades de partida con la que los estudiantes llegan al sistema educativo. No se han identificado políticas de provisión de útiles o libros para el nivel secundario, tampoco de otro tipo de recursos. Las estrategias identificadas en Perú se inscriben en una autonomía parcial, con espacios para el trabajo de cada institución educativa adaptado a su realidad. Podría afirmarse que en Perú existe un desarrollo aún muy incipiente de políticas que buscan el acompañamiento de las trayectorias escolares, muy por el contrario, hay un fuerte énfasis puesto en la calidad educativa a través de diferentes programas. El proyecto de Jornada Escolar Completa aparece como la iniciativa principal pero su implementación parcial y aún en curso no permite evaluar todavía sus resultados.

\section{Paraguay: intervenciones aisladas y un incipiente debate sobre las trayectorias escolares}

En el caso de Paraguay, una política central desarrollada recientemente por el Estado para la inclusión educativa ha sido la gratuidad escolar. Hasta el año 2010, en Paraguay, el Estado garantizaba únicamente la gratuidad de la educación escolar básica (1ํal 9ํgrado). Recién en 2010 se estableció la gratuidad de la educación media. Esto ha significado un profundo impulso a la concreción del Derecho a la Educación de los jóvenes y los adolescentes paraguayos. La Ley 4088/10 establece la obligatoriedad y gratuidad de la educación inicial y la educación media en los establecimientos públicos, garantizando el derecho a una educación integral, permanente y equitativa (Ortiz, 2014).

Junto con la obligatoriedad y gratuidad escolar se pusieron en marcha programas de apoyo a la gratuidad de la educación en Paraguay. Entre ellos se destacan el Programa de Complemento Nutricional -para asegurar una base mínima alimentaria- y el Programa de Útiles Escolares, para todos los niveles del sistema educativo, que propone asegurar los medios e insumos para el trabajo escolar. Se espera que los programas de apoyo a la gratuidad sirvan de atracción y retención en el sistema educativo (Ortiz, 2014). Posteriormente se agrega otra intervención en pos de eximir de costos familiares: la posibilidad de gestionar boletos gratuitos en el transporte público. Un aspecto importante a destacar es que, si bien estas estrategias de provisión directa de recursos a los estudiantes se desarrollan en todo el país, el Estado Nacional está a cargo solo de las acciones desarrolladas en las instituciones educativas de Asunción y el resto es responsabilidad de los gobiernos locales, lo cual puede generar disparidades vinculadas a las desigualdades económicas entre jurisdicciones.

Otro cambio reciente en la educación media en Paraguay se basa en modificaciones en el régimen académico: se instaló en el último curso de la escuela secundaria la evaluación por proyecto (es decir, el desarrollo de un proyecto educativo que ponga en juego las competencias desarrolladas por cada estudiante durante su paso por la escolaridad). Otro dispositivo que complementa esta transformación curricular son las denominadas "clases de retroalimentación", espacios para la recuperación de contenidos para aquellos estudiantes que no han aprobado en tiempo y forma las evaluaciones realizadas.

Es particularmente de interés para dar cuenta del sostenimiento de las trayectorias escolares en las escuelas paraguayas, la función de los profesores guías. El rol del Profesor guía resulta estratégico como núcleo articulador de las interacciones educativas; ejerciendo la mediación en las relaciones internas entre estudiantes y docentes y directivos- y externas de la institución -entre docentes y directivos y la familia- (Méndez, 2008).

Un último aspecto a destacar para el caso de Paraguay es el denominado Programa de Nivelación de los Aprendizajes para estudiantes con sobreedad. El objetivo de esta línea de acción es ofrecer oportunidades educativas con calidad a estudiantes con rezago escolar a partir de la generación de una estrategia con acciones temporales para erradicar la sobre edad y acciones preventivas para combatir la repitencia y la deserción escolar. Para nivelar a los estudiantes se ha desarrollado un programa de competencias básicas a partir de materiales bilingües para estudiantes y materiales para docentes (documentos, guías y material fungible). Esta estrategia genera una alteración en la organización del tiempo escolar y genera un nuevo espacio de formación para la aceleración de las trayectorias demanda la selección de las capacidades fundamentales para trabajar durante el período. 
REVISTA DE LA ESCUELA DE CIENCIAS DE LA EDUCACIÓN, AÑO 16, NRO. 15, VOL. 1, ENERO A JUNIO DE 2020. PÁGINAS 84 - 92 . ISSN $2362-3349$ (EN LÍNEA). POLÍTICAS PARA EL ANDAMIAJE DE TRAYECTORIAS DE ESTUDIANTES EN LA ESCUELA SECUNDARIA EN LATINOAMÉRICA: ESTRATEGIAS PARA GARANTIZAR EL DERECHO A LA EDUCACIÓN EN SOCIEDADES DESIGUALES. PABLO DANIEL GARCíA.

Las estrategias relevadas para la mejora de las trayectorias escolares en Paraguay son escasas. Existen apenas algunos aportes de recursos para los estudiantes, un dispositivo de acompañamiento para los estudiantes en su paso por el nivel secundario y algunas estrategias de innovación pedagógica con un desarrollo aún en construcción. Por ello, la situación de Paraguay en relación a la búsqueda de la mejora para las trayectorias escolares del nivel secundario podría caracterizarse como en gestación, con algunas intervenciones de innovación pedagógicas aisladas que le han permitido empezar a contener la repitencia y desarrollar acciones sistémicas de acompañamiento de los estudiantes durante su paso por el nivel secundario. En este sentido, la labor de los profesores guía (y su formación específica para la orientación de estudiantes) resulta crucial en las instituciones educativas contemporáneas. La cobertura universal de la enseñanza se volvió un imperativo de la política pública durante la reforma educativa al igual que la promoción de la calidad, aunque en los hechos el público concurrente de los sectores desfavorecidos quedó librado a su suerte, bajo el supuesto arbitraje de los méritos (Ortiz, 2014). Este es uno de los problemas principales del sistema educativo paraguayo que, a pesar de los cambios institucionales, curriculares y pedagógicos, mantiene una diferenciación escolar entre sectores sociales, demostrándose que el acceso educativo no es lo mismo que las condiciones sociales de acceso, es decir, la igualdad de oportunidades en el acceso se vuelve desigualdad de resultados en el desempeño (Ortiz, 2014).

\section{Uruguay y su apuesta al tiempo extraescolar como estrategia de retención}

Con miras a desarrollar un sistema educativo inclusivo, en Uruguay se han planificado y desplegado diversas estrategias que buscan atender las trayectorias escolares reales de los estudiantes en el nivel medio. El sistema educativo en Uruguay se caracterizó desde principios del siglo XX por ser altamente inclusivo dado su carácter laico y gratuito (Barcos, 2007). Desde su nacimiento a fines del siglo XIX hasta 1935 que se establece como un ente autónomo del Estado, la Enseñanza Secundaria estuvo bajo la jurisdicción de la Universidad de la República. Y a partir de esa fecha es administrada por el Consejo Educación Secundaria (Barcos, 2007). Por otra parte, hasta el año 1972 la obligatoriedad de la educación estaba dada por los 6 años de la escolaridad primaria. En ese año, se agregan 3 años, luego durante los años de la dictadura se extiende la obligatoriedad por 12 años, pero con una reducción del gasto en educación y la destitución de cientos maestros y profesores y se inicia la implementación un ciclo básico de carácter obligatorio (Barcos, 2007).

En lo que respecta a las estrategias identificadas para la mejora de las trayectorias escolares en el trabajo de campo realizado en Uruguay, pueden considerarse al menos cuatro tipos de intervenciones asociadas a estrategias de provisión directa de recursos a los estudiantes: el Programa de Alimentación Escolar, el Programa de boletos estudiantiles, el Plan Ceibal y el Programa Compromiso Educativo. A partir del 2007 se puso en práctica un plan de cobertura universal en la enseñanza pública, con la aspiración de asistir al proceso de aprendizaje a partir de un uso intensivo de estas tecnologías y de eliminar la desigualdad de acceso a ellas en los niños del país. A este plan se lo denominó Plan Ceibal Plan de Conectividad Educativa de Informática Básica para el Aprendizaje en Línea- (Rivoir y Lamschtein, 2012). La laptop que se brinda a los estudiantes fue especialmente diseñada para estudiantes, quienes son los dueños de la computadora y, por tanto, lo llevan consigo a sus hogares. Esta experiencia significó otra apuesta a la innovación, ya que no solo lo usan en el aula, sino también fuera de ella. En su lanzamiento oficial fue presentado como una iniciativa para la inclusión social, orientada a facilitar el acceso a la información computarizada y el trabajo en red entre los hogares, así como entre estos y los maestros y escuelas (Rivoir y Lamschtein, 2012). Su alcance prácticamente universal en la educación pública fue uno de sus mayores logros y lo transformó en un plan de referencia para la región (Rivoir y Lamschtein, 2012).

Por otra parte, el Programa Compromiso Educativo (PCE) constituye una política iniciada por la administración del Presidente Mujica en 2011. Su objetivo es aumentar la probabilidad de finalización de la educación media. Al igual que otros programas en Uruguay, es de tipo interinstitucional y su diseño incluye tanto incentivos extrínsecos como intrínsecos a través de un componente de transferencia monetaria condicionada (beca), un acuerdo educativo entre el beneficiario y los términos del programa, y un componente de referencia entre pares (Fernández y Pereda, 2010). El PCE, a diferencia de otros programas de becas, posee un acuerdo educativo, que supone un plan consensuado de seguimiento particular a cada uno de los becarios. Este plan pretende ser un instrumento de monitoreo de la integración del estudiante en los distintos espacios (aula, centro educativo y espacio de referencia), así como asistencia del estudiante y participación en las diversas actividades curriculares y no curriculares (DINEM, 2013). Por otra parte, el "Espacio de referencia entre pares" es probablemente el componente más novedoso en compromiso y ha sido definido como instancias de encuentro y aprendizaje, donde un grupo de estudiantes avanzados, acompañan y apuntalan a estudiantes noveles. Estos espacios buscan avanzar en la filiación -en términos de Castel (1995)- del estudiante en la institución. Permiten una comprensión más integral de la problemática de los distintos estudiantes que reciben beca, y esto puede llegar a movilizar otro tipo de apoyos que van más allá de los componentes formales del programa (Fernández y Pereda, 2010).

Uruguay avanzó en el desarrollo con estrategias de innovación pedagógica. Específicamente en este punto, resulta de interés destacar dos programas: el Programa de Liceos con Tutorías y el Programa de Tiempo Extendido. En relación con el Programa "Liceos con Tutorías", surge a partir de un programa antecedente, el "Programa de Impulso a la Universalización" (PIU) que el gobierno de Uruguay puso en marcha en 2008 con el fin de atender a los estudiantes en mayor riesgo de fracaso para la mejora de sus 
trayectorias de los estudios secundarios. Se focalizó en los liceos con los resultados más críticos. Luego el programa cambió su nombre a "Proyecto Liceos con horas de tutorías". Bajo este nuevo formato, el programa tiene el doble objetivo de mejorar los resultados educativos en los centros de ciclo básico que presentan mayores índices de repetición y desvinculación, y apoyar a los docentes en la búsqueda de estrategias que permitan adecuar la enseñanza a la realidad de los jóvenes (INEED, 2016). Consiste en tutorías por asignatura para los estudiantes con más dificultades educativas. Además, en cada centro que participa del programa se designa un profesor coordinador pedagógico, se realiza una instancia mensual de coordinación de todo el equipo docente del centro y hay partidas monetarias para alimentación (meriendas), útiles escolares y vestimenta.

Por otra parte, el Programa Tiempo Extendido (PTE) habilita una serie de innovaciones en el marco de las prácticas pedagógicas de los liceos uruguayos. EI PTE apuesta a promover la educación como un proceso integral que vincula la educación formal con la no formal, al liceo con la comunidad, a través de una propuesta pedagógica abierta, construyendo y consolidando una red de interacciones entre el centro educativo y su entorno. La concreción efectiva del Derecho a la Educación supone reconocer que cada sujeto es único en su trayectoria de aprendizaje y por ello es necesario atender a la multiplicidad de situaciones y propender al planteo de una diversidad de propuestas educativas. La propuesta de tiempo extendido para los liceos, extiende el tiempo pedagógico y resignifica los espacios educativos que buscan ofrecerle a los adolescentes y jóvenes oportunidades para su formación integral. También se plantea la necesidad de trabajar en duplas o tríos docentes desde la interdisciplinariedad y el trabajo colaborativo (Rodríguez, 2014). En los liceos de tiempo extendido el currículum se organiza en núcleos de saberes que superen los límites de las asignaturas. A la vez, se contempla la diversidad de los estudiantes ofreciéndoles diferentes dispositivos de enseñanza y aprendizaje que atiendan edades, contextos institucionales y materiales educativos específicos. Los talleres promueven la producción creativa, la práctica deportiva, el acceso a las más variadas manifestaciones de la cultura, a la acción solidaria, entre otras.

En relación con las estrategias de innovación en la gestión educativa desarrolladas en Uruguay merece una mención el Programa de Tránsito Educativo. Se trata de un programa que surgió en el año 2011 y supone un complejo entramado institucional con el objetivo de lograr que todos los egresados de educación primaria tengan una transición exitosa a la educación media básica, que se inscriban y continúen sus estudios (INEEd, 2016). Opera por zonas con equipos de trabajo conformados por maestros y profesores quienes trabajan con los estudiantes y los equipos permanentes de los centros de origen y de destino. Originalmente tenían prioridad las escuelas de los quintiles más bajos de la población, pero a partir del 2013 se incorporaron todos los liceos con el Proyecto Tutorías (INEEd, 2016). Si bien continúa siendo una política focalizada, su población objetivo se extendió. El Proyecto Tránsito Educativo pone su foco de atención en el pasaje entre los subsistemas que componen el sistema educativo, en las diferencias que cada subsistema presenta respecto a las estrategias didácticas (incluidos los dispositivos de evaluación de aprendizajes y los de disciplina escolar); la organización del tiempo escolar y el tiempo pedagógico; el código curricular (organización curricular y la relación pedagógica). Estos factores pueden constituir puntos críticos de la continuidad y de la transición entre ciclos (Schenck y otros, 2014).

Puede sintetizarse la situación de Uruguay en torno al sostenimiento de las trayectorias escolares como basada en nuevos formatos de la organización del tiempo y el espacio escolar, con una apuesta fuerte a las tutorías y al espacio extraescolar que conforman los talleres como factores de retención. En términos de universalismo-focalización de políticas, se nota una alta preminencia de intervenciones con intención de alcance universal y que a la vez dan autonomía a las instituciones para la construcción de sus propuestas específicas, en el marco de la regulación central.

\section{A modo de cierre}

El análisis de las estrategias desarrolladas por cada uno de los países para la mejora de las trayectorias escolares de los estudiantes en el nivel medio (y particularmente de aquellos que provienen de contextos de vulnerabilidad social) permite dar cuenta de diferentes tipos de abordaje para el logro de este objetivo. En mayor o menor medida, todos los países visitados están trabajando en la transformación de la escuela secundaria y la preocupación por la mejora de las trayectorias escolares aparece en el horizonte como meta. Chile, con su trabajo focalizado en liceos prioritarios, Bolivia con la recuperación del ideario de Warisata, Perú con la propuesta de la Jornada Escolar Completa, Paraguay con la reciente sanción de la gratuidad del nivel medio y Uruguay con los liceos de tiempo extendido, por mencionar solo algunos ejemplos de las intervenciones más destacadas, en todos los casos aparece la necesidad del replanteo del para qué y para quienes del nivel secundario.

El pasaje de una escuela secundaria pensada para las elites y que preparaba casi en exclusividad para el nivel superior a una institución que se abre a toda la población y que forma parte de la escolaridad obligatoria (y por lo tanto que debe "hacer lugar" a todos) conlleva a un profundo replanteo del funcionamiento del nivel secundario. Uno de los aspectos más interesantes de este cambio de modelo radica en el cuestionamiento al propio modo de funcionamiento tradicional expulsivo y excluyente y la búsqueda de alternativas que permitan la generación de otro tipo de experiencias escolares. Se trata de instituciones abiertas a la diversidad del "nuevo público", que antes ni siquiera llegaba al nivel y ahora la meta no solo es que llegue, sino que lo culmine y construya las competencias básicas para su inclusión en un mundo cada vez más complejo y que demanda nuevos tipos de relaciones con el conocimiento. 
REVISTA DE LA ESCUELA DE CIENCIAS DE LA EDUCACIÓN, AÑO 16, NRO. 15, VOL. 1, ENERO A JUNIO DE 2020. PÁGINAS 84 - 92 . ISSN $2362-3349$ (EN LÍNEA). POLÍTICAS PARA EL ANDAMIAJE DE TRAYECTORIAS DE ESTUDIANTES EN LA ESCUELA SECUNDARIA EN LATINOAMÉRICA: ESTRATEGIAS PARA GARANTIZAR EL DERECHO A LA EDUCACIÓN EN SOCIEDADES DESIGUALES. PABLO DANIEL GARCÍA.

La escuela secundaria se está transformando en Latinoamérica y aunque todavía no resulta posible evaluar completamente sus efectos (dado el largo plazo que lleva el estudio de los cambios educativos), es posible afirmar que está en construcción una escuela incluyente que busca superar su formato original elitista y pensado para unos pocos. En esta reconfiguración, un primer punto a destacar es que los propios sistemas educativos están pensando en sus fallas y sus barreras excluyentes.

Referencias bibliográficas

Achili, E. (1992). Las lógicas de la investigación social (mimeo). Rosario: Escuela de Antropología, Universidad Nacional de Rosario.

Barcos, R. (2007). La inclusión educativa en el Uruguay. Avances y desafíos. Presentado en: Taller Regional Preparatorio sobre Educación Inclusiva América Latina, Regiones Andina y Cono Sur Buenos Aires, Argentina, 12 - 14 de septiembre 2007. UNESCO.

Cajías de la Vega, B. (2013). Las propuestas de cambio educativo en Bolivia (1994-2010). En: Revista Ciencia y Cultura. 2013, Número 30, 9 -33.

Castel, R. (1995). La metamorfosis de la cuestión social. Una crónica del salariado. Buenos Aires: Paidós.

Efron, L. (2013). Reformas educativas en el Estado Plurinacional Boliviano: La revalorización de la experiencia de Warisata para la constitución de nuevas ciudadanías. Clío \& Asociados (17), 219-230. En Memoria Académica. Disponible en: http://www.memoria.fahce.unlp.edu.ar/art revistas/pr.6216/pr.6216.pdf

Felfeberg, M y Gluz, N. (2011). Las políticas educativas en Argentina: Herencias de los '90, Contradicciones y Tendencias De "Nuevo Signo. Publicada en: Educ. Soc., Campinas, v. 32, n. 115, p. 339-356, abr.-jun. 2011.

Fernández Lamarra, N. (2006). Reflexiones sobre la planificación de la educación en la Argentina y en América Latina. Evolución, crisis, desafíos y perspectivas. En: Fernández Lamarra, N. y otros (2006). Política, planificación y Gestión de la Educación. Modelos de Simulación en Argentina. Buenos Aires: EDUNTREF.

Fernández, T. y Pereda, C. (2010). Panorama de las políticas e inclusión educativa en la Educación Media y Superior (2005-2009). En: Fernández, T. (2010). La desafiliación en la Educación Media y Superior de Uruguay. Conceptos, estudios y políticas. Montevideo: Universidad de la República / Comisión Sectorial de Investigación Científica.

Gajardo, M. (1999). Reformas educativas en América Latina. Balance de una década. Santiago de Chile: PREAL.

INEEd (2016). Panorama de los programas educativos en 2012 y 2014. Montevideo: INEEd.

Jacinto, C. y Freytes Frey, A. (2004). Políticas y estrategias para el mejoramiento de las trayectorias escolares de los jóvenes. Estudio en la Ciudad de Buenos Aires. Buenos Aires: IIPE UNESCO

Kachinovsky, A. y Gabbiani, B. (2013). Una alternativa al fracaso escolar Hablemos de buenas prácticas. Montevideo: Universidad Nacional de la República, Ediciones Universitarias.

Kaplan, C. (2006). La experiencia escolar inclusiva como respuesta a la exclusión. Consultado en: http://cedoc.infd.edu.ar/upload/la experiencia escolar.pdf

Mamani Cussy, O. (2011) La Educación Comunitaria: su incidencia en la escuela y comunidad. En: Revista Integra Educativa. Vol. IV / № 2, 197-203.

Méndez, O. (2008). El rol del Profesor-Guía Orientador. Asunción: Editorial Don Bosco.

Ortiz, L. (2014). Las políticas de inclusión educativa en Paraguay. Alcances y límites de los programas de gratuidad en educación en la reducción de las desigualdades. En: La educación en su entorno. Sistema educativo y políticas públicas en Paraguay. Asunción: ILAIPP CADEP.

Rivoir, A.L. y Lamschtein, S. (2012). Cinco años del Plan Ceibal: algo más que una computadora para cada niño. Montevideo: UNICEF.

Rodríguez, F. (2014). La co-enseñanza, una estrategia para el mejoramiento educativo y la inclusión. En: Revista latinoamericana de educación inclusiva. ISSN 0718-5480, Vol. 8, № 2, septiembre 2014 - febrero 2015, 219233.

Schenck, M.; Severino, R.; Piriz Bussel, S.; Burguez, S. y Gonzalez, V. (2013). Informe sobre el Plan Tránsito entre Ciclos Educativos. Montevideo: ANEP.

Serulnikov, A. (2008). Políticas públicas de inclusión: entre el gobierno de la educación y las escuelas. Reflexiones a partir de la documentación narrada de experiencias de inclusión. OEA, Proyecto Hemisférico «Elaboración de Políticas y Estrategias para la prevención del Fracaso Escolar», subregión Mercosur.

Strauss, A. y Corbin, J. (1991). Basics of qualitative research. London - New Delhi: Sage.

Terigi, F. (2007). Los desafíos que plantean las trayectorias escolares. Ponencia presentada en III Foro Latinoamericano de Educación Jóvenes y docentes. La escuela secundaria en el mundo de hoy. 28, 29 y 30 de mayo de 2007. Mimeo.

Terigi, F. (2009). Las trayectorias escolares, del problema individual al desafío de política educativa. Buenos Aires: Ministerio de Educación de la Nación.

Terigi, F. y Baquero, R. (1997). Repensando o fracaso escolar pela perspectiva psicoeducativa. En: Abramovicz, A. y Moll, J. (Comps.). Para além do fracasso escolar. Porto Alegre: Papyrus. 